\title{
Exponential decay of correlation for the Stochastic Process associated to the Entropy Penalized Method
}

\author{
Diogo A. Gomes ${ }^{1}$ \\ IST - Portugal \\ Artur O. Lopes ${ }^{2}$ \\ Inst. Mat - UFRGS -Brasil
}

\begin{abstract}
In this paper we present an upper bound for the decay of correlation for the stationary stochastic process associated with the Entropy Penalized Method. Let $L(x, v): \mathbb{T}^{n} \times \mathbb{R}^{n} \rightarrow \mathbb{R}$ be a $C^{1}$ Lagrangian of the form$$
L(x, v)=\frac{1}{2}|v|^{2}-U(x)+\langle P, v\rangle .
$$

We point out that we do not assume more differentiability of $L$ according the the dimension of the torus $\mathbb{T}^{n}$.
\end{abstract}

\section{Definitions and the set up of the problem}

Let $\mathbb{T}^{n}$ be the $n$-dimensional torus. In this paper we assume that the Lagrangian, $L(x, v): \mathbb{T}^{n} \times \mathbb{R}^{N} \rightarrow \mathbb{R}$ has the form

$$
L(x, v)=\frac{1}{2}|v|^{2}-U(x)+\langle P, v\rangle,
$$

where $U \in C^{1}\left(\mathbb{T}^{n}\right)$, and $P \in \mathbb{R}^{n}$ is constant.

\footnotetext{
${ }^{1}$ Partially supported by the Center for Mathematical Analysis, Geometry and Dynamical Systems through FCT Program POCTI/FEDER and also by grant POCI/FEDER/MAT/55745/2004.

2 Partially, supported by CNPq, PRONEX - Sistemas Dinâmicos, Instituto do Milênio, and beneficiary of CAPES financial support.
} 
We consider here the discrete time Aubry-Mather problem [4 and the Entropy Penalized Mather method which provides a way to obtain approximations by continuous densities of the Aubry-Mather measure. We refer the reader to [4] and the last section of [5] for some of the main properties of Aubry-Mather measures, subactions, Peierl's barrier, etc...

The Entropy Penalized Mather problem (see [6] for general properties of this problem) can be used to approximate Mather measures 2 by means of absolutely continuous densities $\mu_{\epsilon, h}(x)$, when $\epsilon, h \rightarrow 0$, both in the continuous case or in the discrete case. In [5] it is presented a Large Deviation principle associated to this procedure. We briefly mention some definitions and results.

Consider, for each value of $\epsilon$ and $h$, the operators acting on continuous functions $\phi$ :

$$
\mathcal{G}[\phi](x):=-\epsilon h \ln \left[\int_{\mathbb{R}^{N}} e^{-\frac{h L(x, v)+\phi(x+h v)}{\epsilon h}} d v\right]
$$

and

$$
\overline{\mathcal{G}}[\phi](x):=-\epsilon h \ln \left[\int_{\mathbb{R}^{N}} e^{-\frac{h L(x-h v, v)+\phi(x-h v)}{\epsilon h}} d v\right] .
$$

Denote by $\phi_{\epsilon, h}$ the solution of $\mathcal{G}\left[\phi_{\epsilon, h}\right]=\phi_{\epsilon, h}+\lambda_{\epsilon, h}$, and by $\bar{\phi}_{\epsilon, h}$ the solution of $\overline{\mathcal{G}}\left[\phi_{\epsilon, h}\right]=\bar{\phi}_{\epsilon, h}+\lambda_{\epsilon, h}$. Let

$$
\theta_{\epsilon, h}(x)=e^{-\frac{\bar{\phi}_{\epsilon, h}(x)+\phi_{\epsilon, h}(x)}{\epsilon h}}
$$

By adding a suitable constant to $\phi_{\epsilon, h}$ or $\bar{\phi}_{\epsilon, h}$, we can assume that $\theta_{\epsilon, h}(x)$ is a probability density on $\mathbb{T}^{N}$. From D. Gomes and E. Valdinoci, it is known that the probability measure on $\mathbb{T}^{N} \times \mathbb{R}^{N}$

$$
\mu_{\epsilon, h}(x, v)=\theta_{\epsilon, h}(x) e^{-\frac{h L(x, v)+\phi_{\epsilon, h}(x+h v)-\phi_{\epsilon, h}(x)-\lambda_{\epsilon, h}}{\epsilon h}},
$$

is a solution to the entropy penalized Mather problem:

$$
\min _{\mathcal{M}_{h}}\left\{\int_{\mathbb{T}^{N} \times \mathbb{R}^{N}} L(x, v) d \mu(x, v)+\epsilon S[\mu]\right\},
$$

where the entropy $S$ is given by

$$
S[\mu]=\int_{\mathbb{T}^{N} \times \mathbb{R}^{N}} \mu(x, v) \ln \frac{\mu(x, v)}{\int_{\mathbb{R}^{N}} \mu(x, w) d w} d x d v,
$$

and

$$
\mathcal{M}_{h}:=\left\{\mu \in \mathcal{M} ; \int_{\mathbb{T}^{N} \times \mathbb{R}^{N}} \varphi(x+h v)-\varphi(x) d \mu=0, \forall \varphi \in C\left(\mathbb{T}^{N}\right)\right\} .
$$


Here $\mathcal{M}$ denotes the set of probability densities on $\mathbb{T}^{N} \times \mathbb{R}^{N}$ and we will call $\mu \in \mathcal{M}_{h}$ a holonomic probability measure.

We will be interested in measures that minimize the functional bellow (under the holonomic constrain)

$$
\int_{\mathbb{T}^{N} \times \mathbb{R}^{N}} L(x, v) d \mu(x, v)+\epsilon S[\mu] .
$$

Note that, for any probability $\mu(x, v)$ by concavity of $\ln$ implies

$$
\begin{gathered}
-S[\mu]=\int_{\mathbb{T}^{N} \times \mathbb{R}^{N}} \mu(x, v) \ln \frac{\int_{\mathbb{R}^{N}} \mu(x, w) d w}{\mu(x, v)} d x d v \leq \\
\ln \int_{\mathbb{T}^{N} \times \mathbb{R}^{N}} \mu(x, v) \frac{\int_{\mathbb{R}^{N}} \mu(x, w) d w}{\mu(x, v)} d x d v=0 .
\end{gathered}
$$

This is the entropy penalized version of the discrete time Aubry-Mather problem, see [4], where we look for probability measures $\mu \in \mathcal{M}_{h}$ that minimize the action

$$
\int_{\mathbb{T}^{N} \times \mathbb{R}^{N}} L(x, v) d \mu(x, v)
$$

Definition 1: The forward (non-normalized) Perron operator $\mathcal{L}$ is defined

$$
x \rightarrow \varphi(x) \Rightarrow x \rightarrow \mathcal{L}(\varphi)(x)=\int e^{-\frac{L(x, v)}{\epsilon}} \varphi(x+h v) d v
$$

In [6] it is shown that $\mathcal{L}$ has a unique eigenfunction $e^{-\frac{\phi_{\epsilon, h}}{h \epsilon}}$ with eigenvalue $e^{-\frac{\lambda_{\epsilon, h}}{h \epsilon}}$

Definition 2: The backward operator $\mathcal{N}$ is given by

$$
x \rightarrow \varphi(x) \Rightarrow x \rightarrow \mathcal{N}(\varphi)(x)=\int e^{-\frac{L(x-h v, v)}{\epsilon}} \varphi(x-h v) d v,
$$

In [6] it is shown that $\mathcal{N}$ has a unique eigenfunction $e^{-\frac{\bar{\phi}_{\epsilon, h}}{h \epsilon}}$ with eigenvalue $e^{-\frac{\lambda_{\epsilon, h}}{h \epsilon}}$

Definition 3: The operator

$$
g(x) \rightarrow \mathcal{F}(g)(x)=\int e^{-\frac{h L(x, v)+\phi_{\epsilon, h}(x+h v)-\phi_{\epsilon, h}(x)-\lambda_{\epsilon, h}}{\epsilon h}} g(x+h v) d v,
$$

is the normalized forward Perron operator. 
From [6] we have that given a continuous function $g: \mathbb{T}^{n} \rightarrow \mathbb{R}$, then $\mathcal{F}^{m}(g)$ converges to the unique eigenfunction $k$ as $m \rightarrow \infty$. We show in this paper that for $\epsilon$ and $h$ fixed, the convergence is exponentially fast.

Our notation:

$$
\begin{gathered}
\theta=\theta_{\epsilon, h}(x)=e^{-\frac{\bar{\phi}_{\epsilon, h}(x)+\phi_{\epsilon, h}(x)}{\epsilon h}}, \\
\gamma(x, v)=\gamma_{\epsilon, h}(x, v)=e^{-\frac{h L(x, v)+\phi_{\epsilon, h}(x+h v)-\phi_{\epsilon, h}(x)-\lambda_{\epsilon, h}}{\epsilon h}},
\end{gathered}
$$

in such way that $\mu_{\epsilon, h}=\theta_{\epsilon, h}(x) \gamma_{\epsilon, h}(x, v)$.

\section{Reversed Markov Process and Adjoint Operator}

In this section we define the reversed Markov process and compute the adjoint of $\mathcal{F}$ in $\mathcal{L}^{2}(\theta)$. We assume $h=1$ from now on.

We can consider the stationary forward Markovian process $X_{n}$ according to the initial probability $\theta(x)$ and transition $\gamma(x, v)$. For example

$$
\begin{gathered}
P\left(X_{0} \in A_{0}\right)=\int_{x \in \mathbb{T}^{n} \cap A_{0}} \theta(x) d x, \\
P\left(X_{0} \in A_{0}, X_{1} \in A_{1}\right)=\int_{x \in \mathbb{T}^{n} \cap A_{0},(x+v) \in A_{1}} \theta(x) \gamma(x, v) d x d v,
\end{gathered}
$$

and so on. Define the backward transfer operator $\mathcal{F}^{*}$ acting on continuous functions $f(x)$ by

$$
\mathcal{F}^{*}(f)(x)=\int \frac{\theta(x-v) \gamma(x-v, v)}{\theta(x)} f(x-v) d v .
$$

The backward transition kernel is given by

$$
Q(x, v)=\frac{\theta(x-v) \gamma(x-v, v)}{\theta(x)} .
$$

The fact that for any $x$ we have $\int Q(x, v) d v=1$ follows from Theorem 32 in [6]. We will show in Corollary 1 that $\theta$ is an invariant measure for the process with transition kernel $Q$, more precisely, that

$$
\int g d \theta=\int \mathcal{F}^{*}(g) d \theta
$$

for any $g \in \mathcal{L}^{2}(d \theta)$.

Theorem 1. $\mathcal{F}^{*}$ is the adjoint of $\mathcal{F}$ in $\mathcal{L}^{2}(\theta)$, that is for all $f, g \in \mathcal{L}^{2}(\theta)$ then

$$
\int f(x) \mathcal{F} g(x) \theta(x) d x=\int g(x) \mathcal{F}^{*} f(x) \theta(x) d x .
$$


Proof. Consider $f, g \in \mathcal{L}^{2}(\theta)$, then

$$
\begin{aligned}
& \int g(x)\left[\mathcal{F}^{*}(f)(x)\right] \theta(x) d x= \\
&=\int g(x)\left[\int \frac{\theta(x-v) \gamma(x-v, v)}{\theta(x)} f(x-v) d v\right] \theta(x) d x \\
&=\int g(x)\left[\int \theta(x-v) \gamma(x-v, v) f(x-v) d v\right] d x \\
&=\int\left[\int[g(x) \theta(x-v) \gamma(x-v, v) f(x-v)] d x\right] d v \\
&=\int\left[\int g(x+v) \theta(x) \gamma(x, v) f(x) d x\right] d v \\
&=\int f(x)\left[\int \gamma(x, v) g(x+v) d v\right] \theta(x) d x \\
&=\int f(x)\left[\int e^{-\frac{L(x, v)+\phi_{\epsilon, 1}(x+v)-\phi_{\epsilon, 1}(x)-\lambda_{\epsilon, 1}}{\epsilon}} g(x+v) d v\right] \theta(x) d x \\
&=\int f(x)[\mathcal{F}(g)(x)] \theta(x) d x,
\end{aligned}
$$

where we use above the change of coordinates $x \rightarrow x-v$ and the fact that $\mu$ is holonomic.

Corollary 1. Consider the inner product $\langle\cdot, \cdot\rangle$ in $\mathcal{L}^{2}(\theta)$. Then $\mathcal{F}$ leaves invariant the orthogonal space to the constant functions: $\{g \mid\langle g, 1\rangle=$ $\left.\int g 1 d \theta=0\right\}$. Furthermore

$$
\int g d \theta=\int \mathcal{F}^{*}(g) d \theta
$$

Proof. Note that $\mathcal{F}(1)=1$, therefore

$$
\int g 1 d \theta=\int g \mathcal{F}(1) d \theta=\int \mathcal{F}^{*}(g) d \theta .
$$

Thus if $\int g 1 d \theta=0$ it follows $\int \mathcal{F}^{*}(g) d \theta=0$.

\section{Spectral gap, exponential convergence and decay of corre- lations}

From [6] it is known that $\mathcal{L}$ has a unique (normalized) eigenfunction $e^{-\frac{\phi_{\epsilon, h}}{h \epsilon}}$ corresponding to the largest eigenvalue $e^{-\frac{\lambda_{\epsilon, h}}{h \epsilon}}$, in the next theorem we prove the this eigenvalue is separated from the rest of the spectrum. 
Theorem 2. The largest eigenvalue of $\mathcal{L}$ is at a positive distance from the rest of the spectrum.

Proof. We will prove the result for the normalized operator

$$
g(x) \rightarrow \mathcal{F}(g)(x)=\int e^{-\frac{h L(x, v)+\phi_{\epsilon, h}(x+h v)-\phi_{\epsilon, h}(x)-\lambda_{\epsilon, h}}{\epsilon h}} g(x+h v) d v .
$$

Recall from [6] that the functions $\phi_{\epsilon, h}(x)$ and $\bar{\phi}_{\epsilon, h}(x)$ are differentiable. In this way we consider a new Lagrangian (adding $\phi_{\epsilon, h}(x+h v)-\phi_{\epsilon, h}(x)-\lambda_{\epsilon, h}$ ) in such way $\mathcal{L}=\mathcal{F}$. We also assume $\epsilon=1$ and $h=1$ from now on.

Therefore,

$$
g(x) \rightarrow \mathcal{F}(g)(x)=\int e^{-L(x, v)} g(x+v) d v,
$$

the eigenvalue is 1 , and, by the results in [6], the corresponding eigenspace is one-dimensional and is generated by the constant functions.

Suppose there exist a sequence of $f_{p} \in \mathcal{L}^{2}(\theta), p \in \mathbb{N}$. such that

$$
\mathcal{F}\left(f_{p}\right)=\lambda_{p}\left(f_{p}\right)
$$

$\left\langle f_{p}, 1\right\rangle=0, \lambda_{p} \rightarrow 1$ and $\left\|f_{p}\right\|=1$. If the operator is compact, then the theorem follows from the classical argument: through a subsequence $f_{p} \rightarrow$ $f$, and since $\lambda_{p} \rightarrow 1$ we have $\mathcal{F}(f)=f$. Furthermore, since $\left\langle f_{p}, 1\right\rangle=0$, it follows $\langle f, 1\rangle=0$, which is a contradiction. Therefore we proceed to establish the compactness of the operator $\mathcal{F}$.

To establish compactness, consider $g \in \mathcal{L}^{2}(\theta)$. We claim that $f=\mathcal{F}(g)$ is in the Sobolev space $\mathcal{H}^{1}$ (see [3] for definition and properties). Indeed, for a fixed $x$, we will compute the derivative of $f$. Integrating by parts we have

$$
\begin{aligned}
\frac{d}{d x} f(x) & =\frac{d}{d x}(\mathcal{F}(g)(x))= \\
& =\int\left(\left[\frac{d}{d x} g(x+v)\right] e^{-L(x, v)}-L(x, v)\left[\frac{d}{d x} e^{-L(x, v)}\right] g(x+v)\right) d v \\
& =\int\left(\left[\frac{d}{d v} g(x+v)\right] e^{-L(x, v)}-L(x, v)\left[\frac{d}{d x} e^{-L(x, v)}\right] g(x+v)\right) d v \\
& =\int\left(\left[\frac{d}{d v} e^{-L(x, v)}\right] g(x+v)-L(x, v)\left[\frac{d}{d x} e^{-L(x, v)}\right] g(x+v)\right) d v \\
& =\int\left(\left[\frac{d}{d v} e^{-L(x, v)}\right]-L(x, v)\left[\frac{d}{d x} e^{-L(x, v)}\right]\right) g(x+v) d v .
\end{aligned}
$$

From the hypothesis about $L$, if $g \in \mathcal{L}^{2}(\theta)$, then indeed $\frac{d}{d x} f$ is also in $\mathcal{L}^{2}(\theta)$ (with the above derivative). 
Note that, for $v$ uniformly in a bounded set

$$
\left\|\frac{d}{d x} f\right\|_{2} \leq\left\|\frac{d}{d x} f\right\|_{\infty} \leq\left\|\left[\frac{d}{d v} e^{-L(x, v)}\right]-L(x, v)\left[\frac{d}{d x} e^{-L(x, v)}\right]\right\|_{2}\|g\|_{2} .
$$

Therefore, $f$ is in the Sobolev space $\mathcal{H}^{1}$.

By iterating the procedure described above, we have that

$$
g_{j}=\mathcal{F}^{j}(g) \in \mathcal{H}^{j} .
$$

It is known that if $j>\frac{n}{2}$, where $n$ is the dimension of the torus $\mathbb{T}^{n}$, then $g_{j}$ is continuous Hölder continuous [3]. Thus the operator $\mathcal{F}$ is compact and $g_{j}$ is differentiable for a much more larger $j$. From the reasoning described before, $f_{p} \rightarrow f$, and $\mathcal{F}(f)=f,\langle f, 1\rangle=0$ and $f$ is differentiable. It is easy to see that the modulus of concavity of $f$ is bounded (the iteration by $\mathcal{F}$ does not decrease it). We can add a constant to $f$ and by linearity of $\mathcal{F}$ we also get a new fixed point for $\mathcal{F}$ (note that $\mathcal{F}(1)=1$ ). Therefore, we can assume $f=e^{-g}$ for some $g$.

In this way, we obtain a contradiction with the uniqueness in Theorem 26 in $[6]$.

Suppose $\int g(x) \theta(x) d x=0$. For $\epsilon, h$ fixed, then it follows from above that $\mathcal{F}^{m}(g) \rightarrow 0$ with exponential velocity (according to the spectral gap).

Consider the backward stationary Markov process $Y_{n}$ according to the transition $Q(x, v)$ and initial probability $\theta$ as above.

Theorem 3. Given $f(x), g(x)$ with $\int f(x) \theta(x) d x=\int g(x) \theta(x) d x=0$, it follows

$$
\int g\left(Y_{0}\right) f\left(Y_{n}\right) d P \rightarrow 0
$$

with exponential velocity.

Proof. Note that

$$
\begin{gathered}
\int g\left(Y_{0}\right) f\left(Y_{1}\right) d P=\int g(x)\left(\int Q(x, v) f(x-v) d v\right) \theta(x) d x= \\
\int g(x)\left[\mathcal{F}^{*}(f)(x)\right] \theta(x) d x=\int f(x)[\mathcal{F}(g)(x)] \theta(x) d x .
\end{gathered}
$$

In the same way, for any $n$

$$
\int g\left(Y_{0}\right) f\left(Y_{n}\right) d P=\int f(x)\left[\mathcal{F}^{n}(g)(x)\right] \theta(x) d x .
$$

The exponential decay of correlation follows from this. 
Theorem 4. Let $f(x), g(x) \in \mathcal{L}^{2}(\theta)$ be such that $\int f(x) \theta(x) d x=$ $\int g(x) \theta(x) d x=0$. Then

$$
\int g\left(X_{0}\right) f\left(X_{n}\right) d P \rightarrow 0
$$

with exponential velocity.

Proof. Now, for analyzing the decay of the forward system, $X_{n}$, with transition $\gamma(x, v)$, we have to consider the backwark operator $\mathcal{F}^{*}$, use the fact that its exponential convergent, that is $\left(\mathcal{F}^{*}\right)^{n}(g) \rightarrow 0$, if $\int g(x) \theta(x) d x=0$, and the result follows in the same way.

\section{References}

[1] Piermarco Cannarsa and Carlo Sinestrari. Semiconcave functions, HamiltonJacobi equations, and optimal control. Progress in Nonlinear Differential Equations and their Applications, 58. Birkhäuser Boston Inc., Boston, MA, 2004.

[2] G Contreras and R. Iturriaga. Global Minimizers of Autonomous Lagrangians. AMS 2004. To appear.

[3] L. C. Evans. Partial Differential equations. AMS 2004. .

[4] D. A. Gomes. Viscosity Solution methods and discrete Aubry-Mather problem. Discrete Contin. Dyn. Syst., 13(1): 103-116, 2005.

[5] D. A. Gomes, A. O. Lopes and J. Mohr. The Mather measure and a Large Deviation Principle for the Entropy Penalized Method, (2007) to appear

[6] Diogo A. Gomes and Enrico Valdinoci. Entropy Penalization Methods for Hamilton-Jacobi Equations. Adv. Math. 215, No. 1, 94-152, 2007. 